\title{
Augmenting the Reality of Phantom Limbs: Three Case Studies Using an Augmented Mirror Box Procedure
}

Deirdre M. Desmond, BA, PhD, Kieran O’Neill, BE, Annraoi De Paor, BE, MS, PhD, Gary McDarby, BE, MSc, PhD, Malcolm MacLachlan, BSc, MSc, MA, PhD

\begin{abstract}
Phantom sensation and phantom pain are common after limb amputation. Previous research documents increased motor control of phantom limbs and alleviation of phantom limb pain through exposure to the "mirror box illusion." This approach centers on the potential for vision and sensorimotor interactions to alter phantom limb perception. The applicability and flexibility of this intervention is limited by methodological constraints inherent in the use of conventional mirrors. This article reports the application of an "augmented reality" intervention that seeks to overcome these constraints. Three case studies are presented, and it is argued that augmented reality technology offers a promising new approach to the investigation of phantom experience and potentially to the treatment of phantom pain. (J Prosthet Orthot. 2006;18:74-79.)
\end{abstract}

KEY INDEXING TERMS: amputation, augmented reality, hand, mirror box, phantom, upper limb

$\mathrm{P}$ ostamputation phantom limb pain persists in the long term for many individuals and may significantly affect quality of life. ${ }^{1-3}$ Clear directives for treatment are not available. ${ }^{4}$ Nonetheless, numerous pain alleviation strategies and treatments ${ }^{5-13}$ have been used to treat phantom pain with varying degrees of success. ${ }^{14}$ Recent research has demonstrated the utility of the noninvasive "mirror box illusion" in investigating phenomenological aspects of embodiment, increasing motor control of phantom limbs, and in some instances, increasing alleviation of phantom limb pain. ${ }^{15-19}$ Premised on the potential for vision and sensorimotor interactions to alter phantom limb perception, this approach uses a visual illusion (the mirror box illusion) to invoke such interaction. ${ }^{17,20}$

DEIRDRE M. DESMOND, BA, PhD, is affiliated with Dublin Psychoprosthetics Group and the Department of Psychology, National University of Ireland, Maynooth, Ireland.

KIERAN O'NEILL, BE, is affiliated with Dublin Psychoprosthetics Group, the Department of Electronic and Electrical Engineering, National University of Ireland, and Rehabilitation Engineering, National Rehabilitation Hospital, Dublin, Ireland.

ANNRAOI DE PAOR, BE, MS, PhD, is affiliated with Dublin Psychoprosthetics Group, the Department of Electronic and Electrical Engineering, National University of Ireland, and Rehabilitation Engineering, National Rehabilitation Hospital, Dublin, Ireland.

GARY MCDARBY, BE, MSc, PhD, is affiliated with Media Lab Europe, Dublin, Ireland.

MALCOLM MACLACHLAN, BSc, MSc, MA, PhD, is affiliated with Dublin Psychoprosthetics Group, and the School of Psychology, University of Dublin, Trinity College, Dublin, Ireland.

Copyright (C) 2006 American Academy of Orthotists and Prosthetists. Correspondence to: Professor Malcolm MacLachlan, Dublin Psychoprosthetics Group, School of Psychology, University of Dublin, Trinity College, Dublin, Ireland; e-mail: malcolm.maclachlan@tcd.ie
By creating a mirror image representation of the missing limb, artificial visual feedback can be generated through apparent transposition of the intact limb's movement to the amputated side. ${ }^{17}$ Viewing the movement of this "virtual limb" while performing synchronous movement tasks with the intact and phantom limbs has been associated with increased motor control over the phantom, and for some individuals, alleviation of phantom pain. ${ }^{15,17,19}$ The beneficial effects of this intervention may relate to correspondence between the feedback provided by the illusory phantom and the actual phantom experience. It has been hypothesized that exposure to such congruence induces plastic changes in the cortical representation of the amputated limb that are associated with pain reduction. ${ }^{21-23}$

There are methodological limitations inherent in the use of conventional mirrors. They necessarily reflect the image of the remaining intact limb; thus, visual feedback is dependent on movement of the intact limb. This limits the potential for experimental manipulation and the realism of the phantom representation. In many cases, the phenomenologically experienced phantom limb differs substantially from the limb before amputation, and from the remaining "intact" limb. ${ }^{24}$ The lack of resemblance between the phantom limb and reflected image may diminish or inhibit the therapeutic value of the intervention. Furthermore, the standard mirror box protocol is inaccessible to those with bilateral amputations or those who experience phantom-like sensation or pain after spinal cord injury.

Virtual and augmented reality technologies in the rehabilitation setting have found promising application in physical rehabilitation, $^{25}$ skills training, ${ }^{26-28}$ pain control, ${ }^{29,30}$ and treatment of psychological disorders, including post-traumatic stress disorder, phobias, and body image disorders. ${ }^{31-35}$ Given the clinical potential and experimental flexibility of such technologies, we sought to extend their application to postamputation phantom phenomena. We developed an "augmented mirror box" to allow artificial visual feedback to be remotely generated 
(i.e., generated independently of contralateral limb movement), thus facilitating presentation of noncontingent phantom limb movement. If phantom pain experience is influenced by contradictory proprioceptive and visual feedback, manipulation of the association between "felt" movements and visually presented movements could have therapeutic potential. This article describes the technological development of the augmented mirror box and initial exploration of the effects of noncontingent phantom limb movement through three case studies.

\section{MATERIALS AND METHODS}

The standard mirror box, described by Ramachandran and colleagues, ${ }^{17,20}$ is constructed by placing a mirror vertically inside an open box. The mirror is aligned along the midline, and the participant places his or her intact arm on the reflective side of the mirror so that when viewed from above the intact arm and its mirror image create the illusion that the amputated limb is also intact.

The augmented mirror box consists of a three-dimensional (3D) graphical representation of an arm on a flat computer screen that is controlled by a wireless data glove (5DT Data Glove 5W [Fifth Dimensions Technologies, Irvine, CA], transmission frequency $433.92 \mathrm{MHz}$.). The data glove, worn on the intact arm, measures finger flexure and the orientation (both pitch and roll) of the user's hand. The information received from the glove is fed back to the computer so that the image on the screen appears to the participant to move in real time. The image is created using a computer graphics software package (3D Studio MAX, Berkeley, CA) and a real-time computer graphics games engine. ${ }^{36}$ Movements of the augmented phantom can thus be controlled by movement of the data glove (i.e., providing a reflection similar to that provided by a standard mirror) or can be remotely controlled via a laptop computer.

Each participant completed a semistructured interview describing the history of amputation, artificial limb use, and phantom experiences. Pain descriptors from the McGill Pain Questionnaire (short form) ${ }^{37}$ were used to elicit the quality of each respondent's phantom pain experience. In the experimental phase of the study, participants completed a series of symmetric and asymmetric arm movements using both their phantom and contralateral arms while wearing a data glove on their intact arm. The movements were comprised of: 1) holding one's hands (phantom and intact) pronate and tapping one's index fingers simultaneously (the tapping task); and 2) attempting to move all fingers simultaneously with the palm of the hand held facing the mirror. Visual feedback was provided via a standard mirror and in subsequent trials presented via a flat computer screen taking the place of the mirror as described above. Given the exploratory nature of the study, the varying levels of volitional control participants had over their phantoms, and their varying reactions to the visual feedback, the task demands were varied across participants to appropriately consider their individual experiences and reactions. Each participant chose to wear his or her prosthesis while completing the trial.

\section{INCLUSION CRITERIA}

Participants had to have a unilateral right-side (necessitated by the use of a left-hand fit data glove) upper limb amputation and be between 18 and 65 years old. Participants were recruited from a national limb-fitting clinic, IDS Ltd., at Cappagh National Orthopaedic Hospital. Three participants were included in this study.

\section{PARTICIPANT 1}

A 40-year-old man underwent right-side transhumeral amputation because of osteogenic sarcoma approximately 3 years before this research. He currently uses a passive cosmetic prosthesis during all waking hours.

After his amputation, participant 1 stated that he felt his arm was still intact and in its normal position. Over 6 to 7 months, he experienced telescoping of his phantom. He reports his phantom hand is now felt to be attached to the end of the residual limb and cramped in a claw-like position, which although discomforting is not painful. He is not aware of temperature changes in the phantom but sometimes experiences changes in pressure, itching between the fingers, and nonpainful tingling. His phantom does not make involuntary movements, and he can produce only tiny movements of the fingers $(<1 \mathrm{~cm})$. The phantom fingers are most vividly experienced, followed by the palm of the hand and then the wrist.

Phantom pain occurs every 3 or 4 hours and lasts less than 10 minutes. It is cramping, sharp, and stabbing in quality. Participant 1's phantom pain proved resistant to treatment with Tegretol (750 mg daily). He achieves pain relief by physically manipulating his residual limb. Emotional stress can trigger episodes of phantom pain.

\section{INTERVENTION}

\section{VISUAL FEEDBACK: STANDARD MIRROR}

When performing the tapping task, participant 1 reported that his phantom became more tense. His phantom forefinger moved slightly but became sore and uncomfortable. His attempts to move all fingers simultaneously also increased tension and nervous discomfort but resulted in greater pain. Participant 1 felt his phantom more vividly when viewing the reflected image.

\section{VISUAL FEEDBACK: AUGMENTED MIRROR BOX}

During the tapping task a similar but much less pronounced effect ensued than when using the standard mirror. Participant 1's attempts to move all fingers simultaneously produced pain, but the sensation was also less intense than when using the mirror.

To further explore the utility of the augmented reality image, the image was manipulated so that two fingers of the virtual phantom hand were frozen (i.e., the image of the fingers 
did not move despite movement of the data glove). The participant then attempted to move all fingers simultaneously as before. He reported increasing strain and tightness in the three moving fingers while the two fingers frozen on the screen became less strained. An additional virtual finger was frozen, and again during the simultaneous finger movement task, the participant reported relaxation of the three frozen phantom fingers and increasing strain and tightness in the two fingers moving on screen. He described this as "strange," "surprising," and "astonishing."

The phantom image was then completely frozen (i.e., none of the visually presented fingers moved, despite attempts to move the phantom fingers and to produce movement of the data glove). When performing the simultaneous movement task, participant 1 reported diminishing tightness in his phantom. After about 10 seconds, the tight sensation extinguished completely and he reported a pleasant and relaxed feeling that he had not previously experienced. Movement of the fingers of the phantom image was then remotely controlled and spontaneously initiated. Under these conditions the tense feeling in the phantom intensified.

The standard mirror box was reintroduced to further investigate these outcomes. The participant viewed the standard mirror image of his intact left arm, with the hand at rest, while attempting to move all the fingers of his phantom hand simultaneously. He reported a strengthening of phantom sensation in all his fingers and a stab of pain in his phantom thumb. The tapping task also intensified the vividness of sensation in his phantom index finger, and he reported stabbing pain in the fingertip. Finally, the participant allowed his phantom to assume the at-rest position of his left hand (and by default the phantom image), producing a relaxed sensation.

\section{SUMMARY}

For participant 1 the presentation of visual feedback using the standard mirror and the augmented mirror altered and intensified phantom experience. This effect was more pronounced when using the standard mirror. The presentation of inconsistent feedback (i.e., presenting the image of the phantom as stationary when the participant was in fact trying to move his phantom fingers) reduced phantom experience, an effect specific to particular fingers. In contrast, in the standard mirror condition, the presentation of inconsistent feedback induced phantom pain. The reason for this discrepancy is unclear. Participant 1 noted that emotional stress could trigger phantom pain. Although it is possible that at the end of the testing session (i.e., when the standard mirror was reintroduced) the participant was tired and frustrated by the task and this induced pain, he did not believe this to be the case.

\section{PARTICIPANT 2}

A 25-year-old man sustained amputation of the right forequarter in a motor vehicle accident approximately 6 years before this research. He uses a passive cosmetic prosthesis for social occasions only "in order to blend in."

Although unable to pinpoint the timing of onset of phantom sensation and phantom pain because of pain medication, participant 2 reported constant phantom limb sensation and intermittent but frequent phantom pain. The phantom pain was resistant to treatment using $100 \mathrm{mg}$ morphine patch Duragesic, applied every 3 days or via ganglion nerve block. Participant 2 previously experimented with the mirror box procedure. Using a large mirror placed down the midline of the body, he concentrated on the reflected image of his intact arm but did not try to move it. The effect was to make the phantom feel "freer," although it did not change in terms of posture.

Participant 2's phantom arm is positioned across his body, raised in a protective pose. He reports that sensation is most vivid in the hand, which is held in a loosely clenched fist with the thumb on the outside. Although he does not feel the forearm and upper arm per se, he is aware of the position of the elbow. His phantom always feels "warm," and he sometimes experiences changes in pressure and tingling. The phantom does not make involuntary movements, and he cannot voluntarily generate movements in his phantom limb.

Participant 2 experiences almost daily phantom sensation in the thumb and forefinger area that he describes as stabbing, hot/burning, and tiring/exhausting. Emotional stress and physical illness can trigger and/or worsen his phantom pain, and pain sometimes occurs when performing tasks requiring particular dexterity with the intact hand.

\section{INTERVENTION}

\section{NO VISUAL FEEDBACK}

Performing the tapping task produced no change in phantom sensation, and the participant was unable to produce movement in the phantom. His attempts to move all fingers simultaneously were also ineffective.

\section{VISUAL FEEDBACK: STANDARD MIRROR}

The participant indicated that simply viewing the reflected image did not produce any change in his phantom. When performing the tapping task, his phantom felt a little "freer," but he was still unable to produce movement. As his phantom hand was held as a clenched fist, he attempted to open and close both intact and phantom fists simultaneously and in synchrony. This had no effect on his phantom. Similarly, his attempts to move all fingers simultaneously were unsuccessful.

\section{VISUAL FEEDBACK: AUGMENTED MIRROR BOX}

Participant 2 reported that performing the same tapping task while viewing the virtual image had no effect on his phantom. His attempts to move all fingers simultaneously were also ineffective.

\section{SUMMARY}

Before testing, participant 2 was unable to produce voluntary movement in his phantom. Providing feedback, whether us- 
ing a standard mirror or the augmented reality box, did not alter volitional control of his phantom.

\section{PARTICIPANT 3}

A 49-year-old woman sustained injuries in a motor vehicle accident and subsequently underwent amputation of her right arm distal to the elbow, approximately 12 years before this research. She wears a prosthesis all day every day, mainly using a cosmetic prosthesis but also using a myoelectric prosthesis for particular tasks.

Participant 3 experienced phantom sensation immediately after amputation surgery. She is not ordinarily aware of sensation in her missing forearm but is aware of her phantom hand. She feels her fingertips touching her thumb and although originally felt in this position, her little finger is now raised. She does not experience her phantom fingers individually but rather as a unit, nor does she experience changes in temperature in her phantom. Changes in pressure and a nonpainful tingling sensation do occur.

When painful, participant 3's phantom hand is in a clenched fist with the nails digging into the palm of the hand. The pain is "shooting" and "stabbing" and can be triggered by emotional stress. She previously sought phantom pain treatment at a pain clinic but was dissatisfied with the drug-based approach. She no longer uses medication for phantom pain because she has "learned to live with it" and the pain has decreased in both frequency and intensity. She can occasionally achieve pain relief by visualizing her hand opening and relaxing.

Participant 3 can generate voluntary movements in her phantom, and, with the exception of her little finger, finger movement is felt in tandem with movement of her myoelectric prosthesis. Following periods of disuse, donning the prosthesis and attempting to "get back into the rhythm of using it" cause distress and pain.

\section{INTERVENTION}

\section{VISUAL FEEDBACK: STANDARD MIRROR}

Simply viewing the reflected image did not produce spontaneous change in participant 3's phantom. When attempting to move all fingers simultaneously, she reported an intensification of tingling sensations that was "very uncomfortable." While performing the tapping task, participant 3 reported that she became very aware of the palm of her phantom hand, an awareness not normally experienced. Although she was attempting to tap only the phantom index finger, the vividness of other areas of her phantom hand also intensified.

\section{NO VISUAL FEEDBACK}

In the absence of visual feedback, performing the tapping task and attempting to simultaneously move all fingers produced the same sensations as in the previous condition. However, the participant remarked that it was more difficult to concentrate and that producing movement in her phantom fingers was easier when viewing the reflected image.

\section{VISUAL FEEDBACK: AUGMENTED MIRROR BOX}

During performance of the tapping task, participant 3 reported increased awareness of sensation in all parts of her phantom hand, in particular in the little finger and palm. Furthermore, she indicated that the motion of her index finger was better than before, but the experience was somewhat uncomfortable. When attempting to move all fingers simultaneously, she reported a more diffuse and "jumbled" pain in her phantom hand. Her hand felt "real tensed up without being specific to any area."

Movement of the fingers of the phantom image was then remotely controlled and spontaneously initiated without verbal cueing. The participant experienced tingling in all of the fingers and became increasingly aware of each finger as it moved. Although always aware of the little finger independently, she indicated "the third and fourth fingers are new"; "I haven't felt that experience before." She reported that this sensation was not unpleasant. Although the participant previously experienced her fingers as a unit, during this procedure she gained control over independent movement of her fingers.

The augmented reality phantom image was then remotely manipulated so that two of the fingers of the phantom hand were frozen (i.e., the image of the fingers did not move despite movement of the data glove on the contralateral hand). The participant attempted to move all her fingers simultaneously as before but reported being able to move only her little finger: the other fingers felt unpleasantly constrained, and the thumb felt as though it were being "pulled." A subsequent modification of the augmented image froze all movement except that of the little finger. When trying to move all fingers simultaneously, the participant reported that her little finger moved, but the other fingers were "straight out but constrained." Moving all fingers simultaneously while viewing an image derived by inverting the earlier manipulation (i.e., only the little finger was frozen) resulted in tingling in all of the phantom fingers, and she reported the "little one [finger] won't move but I can feel each one." She felt her index finger was stiff, but was able to move it with effort. This was a "strange sensation." When movement of the virtual image of the little finger was reinstated but remotely controlled without verbal cueing, the participant felt movement of her phantom finger occurring in tandem with the visually presented image.

\section{VISUAL FEEDBACK: STANDARD MIRROR}

Performing the simultaneous movement task while viewing the standard mirror image, participant 3 reported that her little and ring fingers were moving together as a unit while her index finger was moving independently.

\section{SUMMARY}

For participant 3 visual feedback in the standard mirror and augmented mirror conditions intensified and extended phantom experience. Viewing the augmented reality phantom image facilitated greater movement of her phantom index 
finger and novel independent movement of her third and fourth fingers as independent digits. Such movement had previously been impossible. Movement of the fingers of participant 3's phantom hand could be remotely generated.

\section{DISCUSSION}

The three case studies presented here are the first results to be reported using augmented reality technology with individuals with limb amputation. Although necessarily preliminary, these results suggest that augmented reality technology offers a promising new approach to investigation of phantom experiences and potentially to the treatment of phantom pain. Participants 1 and 3 reported intensification of their phantom experiences through exposure to the augmented reality image. Furthermore, participant 3 reported increased control over her phantom fingers during the augmented reality procedure, and participant 1 reported relaxation and pain reduction during presentation of inconsistent feedback in the augmented reality scenario. Although the standard mirror image was reported to be more compelling by participant 1 , participant 3 reported that the augmented image had a more dramatic effect on her phantom experience. In contrast, participant 2 found that neither the conventional mirror nor the augmented mirror influenced his phantom experience. The individual variability reported here in response to both the standard mirror and the augmented mirror interventions is consistent with findings elsewhere. ${ }^{17,18}$

The unique contribution of the current research is our observation that incongruent movement of the phantom limb, visually fed-back via the augmented reality box, may reduce the perception of discomfort and pain. Such a possibility has previously, by definition, not been possible without augmented reality technology. For participant 1, attempting to move his phantom while viewing an image of a partially frozen hand (i.e., instances in which some but not all of the visually presented fingers were free to move consistent with movement of the data glove) resulted in alleviation of discomfort in the seemingly frozen fingers. This finding is somewhat at odds with the later observation that incongruent feedback provided by the conventional mirror induced phantom pain. This effect clearly warrants additional investigation.

Participant 3's experience with the augmented reality box demonstrates that it is possible to remotely or externally induce movement in a phantom limb. Her phantom fingers were felt to move in tandem with the externally controlled phantom image. This finding has potential clinical utility, suggesting the possibility to free painfully clenched or positioned phantoms through exposure to appropriate visual cues. However, caution is warranted because Giraux and Sirigu ${ }^{21}$ observed that passively exposing individuals with brachial plexus avulsion to prerecorded arm movements could also induce painful phantom experiences.

The substantial limitations of case study methodology have been clearly elucidated elsewhere. ${ }^{38}$ Although the current study afforded opportunity for presentation of an inno- vative technique, additional controlled studies are needed. In particular, investigation of the mechanisms of these changes is necessary. It is possible that the outcomes reported were a consequence of a placebo effect.

Although the initial case studies reported here focused on feedback of noncontingent phantom limb movement, future developments of the technology will be targeted at introducing more phenomenologically authentic phantom limb representations. Such representations, premised on individuals' experience of their phantoms, may incorporate postures and structures not ordinarily experienced and thus not readily reproducible using conventional mirrors. A stronger test of the hypothesis that visual feedback of a "virtual arm" increases awareness and/or controllability of a phantom limb and reduces phantom pain may emerge.

\section{CONCLUSION}

This study represents a first step toward the development of an augmented reality intervention with the potential to treat phantom limb pain by harnessing the therapeutic value of the visual feedback received from the augmented environment. Considerable scope for additional research remains.

\section{ACKNOWLEDGMENTS}

Funded by the Higher Education Authority of Ireland.

The authors thank the Higher Education Authority for funding. The authors also thank Mr. Dave Allen and the staff of IDS Ltd., Dublin, for assistance in participant recruitment; the staff of Media Lab Europe for technical assistance; and the participants for their time and effort in taking part.

\section{REFERENCES}

1. Ehde DM, Czerniecki JM, Smith DG, et al. Chronic phantom sensations, phantom pain, residual limb pain, and other regional pain after lower limb amputation. Arch Phys Med Rehabil 2000;81:1039-1044.

2. Kooijman CM, Dijkstra PU, Geertzen JHB, et al. Phantom pain and phantom sensations in upper limb amputees: an epidemiological study. Pain 2000;87:33-41.

3. Gallagher P, Allen D, MacLachlan M. Phantom limb pain and residual limb pain following lower limb amputation: a descriptive analysis. Disabil Rehabil 2001;23:522-530.

4. Baron R, Wasner G, Lindner V. Optimal treatment of phantom limb pain in the elderly. Drugs Aging 1998;12:361-376.

5. Oakley DA, Whitman LG, Halligan PW. Hypnotic imagery as a treatment for phantom limb pain: two case reports and a review. Clin Rehabil 2002;16:368-377.

6. Leskowitz E. Phantom limb pain treated with Therapeutic Touch: A case report. Arch Phys Med Rehabil 2000;81:522-524.

7. Nikolajsen L, Hansen CL, Nielsen J, et al. The effect of ketamine on phantom pain: a central neuropathic disorder maintained by peripheral input. Pain 1996;67:69-77. 
8. Belleggia G, Birbaumer N. Treatment of phantom limb pain with combined EMG and thermal biofeedback: a case report. Appl Psychophysiol Biofeedback 2001;26:141-146.

9. Lu TV. Acupuncture treatment for phantom limb pain. Alter Ther Health Med 1998;4:124.

10. Rasmussen KG, Rummans TA. Electroconvulsive therapy for phantom limb pain. Pain 2000;85:297-299.

11. Bone M, Critchley P, Buggy DJ. Gabapentin in postamputation phantom limb pain: A randomized, double-blind, placebocontrolled, cross-over study. Region Anesth Pain Med 2002;27: 481-486.

12. Mitchell AC. An unusual case of chronic neuropathic pain responds to an optimum frequency of intravenous ketamine infusions. J Pain Symptom Manage 2001;21:443-446.

13. Rosen G, Willoch F, Bartenstein P, et al. Neurophysiological processes underlying the phantom limb pain experience and the use of hypnosis in its clinical management: an intensive examination of two patients. Int J Clin Exp Hypn 2001;49:38-55.

14. Sherman RA, Sherman CJ, Gall NG. A survey of current phantom limb pain treatment in the United States. Pain 1980;8: 85-99.

15. Brodie EE, Whyte AS, Waller B. Increased motor control of a phantom leg in humans results from the visual feedback of a virtual leg. Neurosci Letters 2003;341:167-169.

16. Hunter JP, Katz J, Davis KD. The effect of tactile and visual sensory inputs on phantom limb awareness. Brain 2003;126: $579-589$

17. Ramachandran VS, Rogers-Ramachandran D. Synaesthesia in phantom limbs induced with mirrors. Proc $R$ Soc Lond $B$ 1996;263:377-386.

18. MacLachlan M, Desmond D, Horgan O. Psychological correlates of illusory body experiences. J Rehabil Res Devel 2003;40:59-66.

19. MacLachlan M, McDonald D, Waloch J. Mirror treatment of lower limb phantom pain: a case study. Disabil Rehabil 2004; 26:901-904.

20. Ramachandran VS, Hirstein W. The perception of phantom limbs. Brain 1998;121:1603-1630.

21. Giraux P, Sirigu A. Illusory movements of the paralyzed limb restore motor cortex activity. NeuroImage 2003;20:S107-S111.

22. Giraux P, Sirigu A, Schneider F, Dubernard JM. Cortical reorganization in motor cortex after graft of both hands. Nature Neurosci 2001;4:691-692.

23. Flor H, Elbert T, Knecht S, et al. Phantom-limb pain as a perceptual correlate of cortical reorganisation following arm amputation. Nature 1995;375:482-484.
24. Wright A. Wellcome Trust Sci Art Project. 1997, http:// www.medphys.ucl.ac.uk/mgi/alexa/alexawright.html.

25. Sveistrup H, McComas J, Thornton M, et al. Experimental studies of virtual reality-delivered compared to conventional exercise programs for rehabilitation. Cyberpsychol Behav 2003; 6:245-249.

26. Harrison A, Derwent G, Enticknap A, et al. The role of virtual reality technology in the assessment and training of inexperienced powered wheelchair users. Disabil Rehabil 2002;24: 599-606.

27. Lee $\mathrm{JH}, \mathrm{Ku} \mathrm{J}$, Cho $\mathrm{W}$, et al. A virtual reality system for the assessment and rehabilitation of the activities of daily living. Cyberpsychol Behav 2003;6:383-388.

28. Gourlay D, Lun KC, Lee YN, Tay J. Virtual reality for relearning daily living skills. Int J Med Inform 2000;60:255-261.

29. Hoffman HG, Patterson DR, Carrougher GJ, et al. The effectiveness of virtual reality pain control with multiple treatments of longer durations: a case study. Int J Human-Computer Interact 2001;13:1-12.

30. Hoffman HG, Garcia-Palacios A, Patterson DR, et al. The effectiveness of virtual reality for dental pain control: a case study. Cyberpsychol Behav 2001;4:527-535.

31. Riva G, Bacchetta M, Baruffi M, Molinari E. Virtual reality-based multidimensional therapy for the treatment of body image disturbances in obesity: a controlled study. Cyberpsychol Behav 2001;4:511-526.

32. Wald J, Taylor S. Efficacy of virtual reality exposure therapy to treat driving phobia: a case report. J Behav Ther Exp Psychiatry 2000;31:249-257.

33. Garcia-Palacios A, Hoffman H, Carlin A, et al. Virtual reality in the treatment of spider phobia: a controlled study. Behav Res Ther 2002;40:983-993.

34. Krijn M, Emmelkamp PMG, Biemond R, et al. Treatment of acrophobia in virtual reality: The role of immersion and presence. Behav Res Ther 2004;42:229-239.

35. Rothbaum BO, Hodges L, Alarcon R, et al. Virtual reality exposure therapy for PTSD Vietnam veterans: a case study. J Traumatic Stress 1999;12:263-271.

36. Eberly D. 3D Game Engine Design: A Practical Approach to Real-Time Computer Graphics. London: Morgan Kaufmann; 2001.

37. Melzack R. The McGill Pain Questionnaire: major properties and scoring methods. Pain 1975;1:277-299.

38. Graziano A, Raulin M. Research Methods: A Process of Inquiry. 5th ed. London: Pearson; 2004.

\section{TO EARN TWO PCE CREDITS:}
1. Read the article on pages 74-79.
2. Go to the On-Learning Center at www.oandp.org. and complete the examination. 